\title{
Hematúria no jovem adulto: um caso de síndrome da junção pielo-ureteral
}

Samuel Gomes ${ }^{1}$

\section{RESUMO}

Introdução: A síndrome da junção pielo-ureteral é uma patologia mais frequente em idade pediátrica. Consiste numa forma de obstrução do trato urinário, que resulta de um bloqueio parcial ou total intermitente do fluxo de urina a nível da junção pielo-ureteral. É a causa mais comum de hidronefrose no período pré-natal e, quanto aos jovens adultos (18 aos 24 anos), a taxa de internamento por esta causa foi de 0,7 por 100.000 no ano 2000 nos Estados Unidos da América.

Descrição do caso: Homem de 21 anos, sem antecedentes pessoais de relevo, que recorreu ao serviço de urgência por quadro de dor referida ao flanco esquerdo e hematúria com início no próprio dia. Foi medicado sintomaticamente e teve alta para o médico de família para estudo de cálculo renal. Recorreu a consulta do centro de saúde passados três dias com manutenção dos sintomas. Ao exame objetivo verificou-se punho-percussão lombar sem alterações bilateralmente e palpação abdominal com massa medindo cerca de 15 por $20 \mathrm{~cm}$, não móvel, de consistência duro-elástica, nos quadrantes esquerdos. Foi encaminhado novamente ao serviço de urgência para reavaliação urgente. A tomografia computorizada urológica revelou bolsas hidronefróticas a substituir o rim esquerdo, sem imagens sugestivas de obstrução, observação compatível com síndrome da junção pielo-ureteral. Internou-se o utente para cateterização ureteral e avaliação funcional do rim esquerdo.

Comentário: A hematúria em contexto de dor unilateral referida ao flanco deve fazer suspeitar em primeiro lugar de nefrolitíase, mas outras causas não devem ser negligenciadas, principalmente em apresentações menos típicas. Este caso reforça a ideia de que uma patologia mais frequente em idade pediátrica pode ser encontrada em doentes adultos. É, no entanto, pouco comum, tendo sido a validação das queixas do utente e o exame objetivo cuidado em consulta de medicina geral e familiar fatores chave para uma orientação adequada.

Palavras-chave: Hematúria; Síndrome da junção pielo-ureteral; Jovem adulto; Hidronefrose.

\section{INTRODUÇÃO}

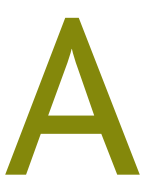

síndrome da junção pielo-ureteral é uma patologia tipicamente pediátrica, de origem congénita ou adquirida. Resulta de uma obstrução do trato urinário ao nível da junção pielo-ureteral, que pode ser parcial ou total intermitente. As causas congénitas podem estar relacionadas com alterações anatómicas (vaso renal inferior acessório ou aberrante, por exemplo) ou com distúrbios funcionais do ureter (como a hipoplasia ureteral). As causas adquiridas incluem a fibrose por manipulação cirúrgica prévia do ureter ou neoplasia..$^{1-4}$ É uma entidade mais frequente no sexo masculino e afeta predominantemente o rim esquerdo, consistindo na causa mais co-

1. Médico Interno de Medicina Geral e Familiar. USF Amato Lusitano, ACeS Amadora, ARS Lisboa e Vale do Tejo. Amadora mum de hidronefrose no período pré-natal. ${ }^{1-2}$ Para isto contribui a maior complacência dos tecidos corporais nesta fase do desenvolvimento, uma vez que possibilitam uma maior acomodação de volume. ${ }^{3}$ Quando não identificada durante a vigilância pré-natal pode manifestar-se na infância, com dor no flanco ou abdominal intermitente, com ou sem náuseas ou vómitos e hematúria. Esta entidade clínica aumenta o risco de dano renal após traumatismos minor e é, ainda, fator de risco para litíase renal e hipertensão nesta faixa etária. ${ }^{1-2} \mathrm{Ou}-$ tras vezes, acaba por ser um achado incidental em exames de imagem realizados por outras razões. ${ }^{1}$

Em Portugal, um estudo com recém-nascidos verificou uma incidência de dilatação pielo-calicial congénita de $2,7 \%$, dos quais apenas $2 \%$ necessitou de correção cirúrgica. ${ }^{5}$ Quanto aos adultos entre os 18 e os 
24 anos de idade verificou-se, nos Estados Unidos da América, uma taxa de internamento por síndrome da junção pielo-ureteral de 0,7 por 100.000 durante o ano 2000. ${ }^{6}$ Não sendo, portanto, frequente nestas faixas etárias, o seu diagnóstico depende de uma correta avaliação clínica do utente sintomático, o que justifica a pertinência da apresentação deste caso.

\section{DESCRIÇÃO DO CASO}

Jovem adulto do sexo masculino, com 21 anos de idade, caucasiano e de nacionalidade brasileira. Vive com a mãe numa estrutura familiar monoparental, tendo imigrado para Portugal aos sete anos. Atualmente é empregado de mesa. À data do caso clínico, o utente não tinha antecedentes patológicos pessoais e familiares relevantes, não tinha medicação crónica prescrita e também não existiam alergias medicamentosas conhecidas.

Recorreu ao serviço de urgência por dor lombar moderada à esquerda, disúria e hematúria macroscópica com algumas horas de evolução. Ao exame objetivo apresentava estabilidade hemodinâmica, apirexia, dor à palpação do flanco abdominal esquerdo e punho-percussão do ângulo costo-vertebral dolorosa ipsilateralmente. Do resultado dos meios complementares de diagnóstico realizados não se objetivaram alterações no hemograma, ionograma e na função renal, sendo que a PCR estava discretamente aumentada $(4,5 \mathrm{mg} / \mathrm{dl})$. A avaliação sumária da urina mostrou hemoglobina positiva (3+), 75 leucócitos por campo e nitritos negativos. Realizou ainda radiografia abdominal antero-posterior em ortostatismo, descrita como não tendo alterações de relevo (Figura 1). Neste contexto, o utente foi tratado sintomaticamente e, após melhoria das queixas álgicas, encaminhado para o seu médico de família para investigação e avaliação de eventual cálculo renal.

Passados três dias, o utente agendou consulta na sua unidade de cuidados de saúde primários dando conta do episódio de urgência e referindo manutenção das queixas. Apurou-se ainda que a dor referida ao flanco esquerdo tinha irradiação lombar, era de intensidade moderada e contínua. Para além de hematúria referiu ainda disúria, polaquiúria, urgência miccional e urina com cheiro fétido. Ao exame objetivo mantinha-se apirético, no entanto sem dor à punho-percussão do ângulo costo-vertebral. Também em contraste com a avaliação prévia em serviço de urgência, a palpação abdo-

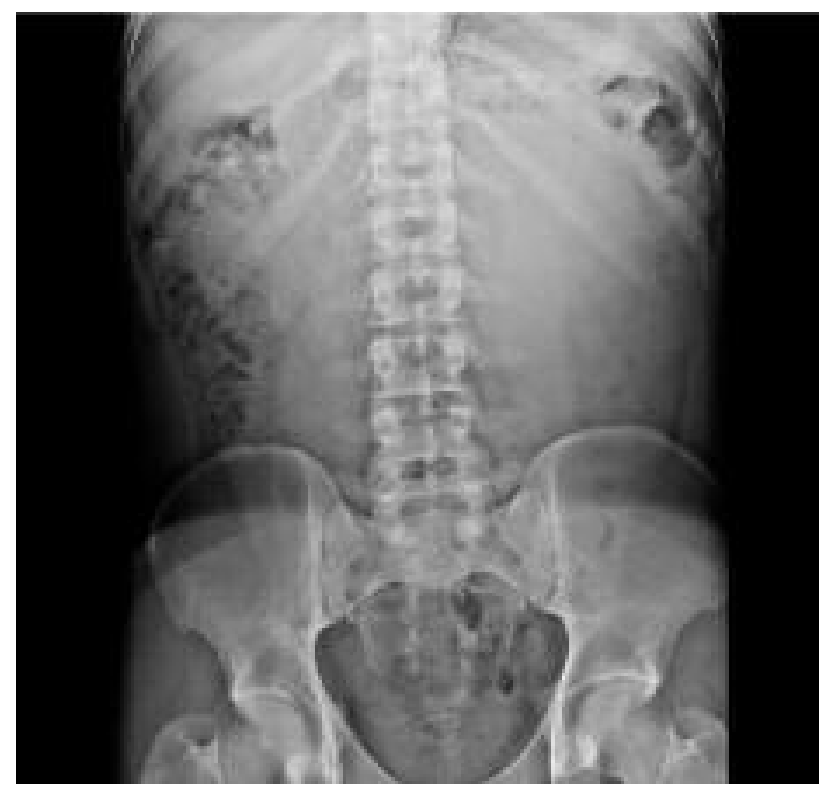

Figura 1. Radiografia abdominal antero-posterior em ortostatismo realizada pelo utente no primeiro episódio de urgência.

minal, para além de dolorosa nos quadrantes esquerdos, permitiu ainda verificar a existência, nessa localização, de uma massa imóvel com cerca de 15 por $20 \mathrm{~cm}$, de consistência duro-elástica e de limites mal definidos. Assim, as características do exame objetivo do abdómen não permitiam excluir claramente a existência de defesa abdominal. Tendo esta avaliação em conta levantaram-se as hipóteses diagnósticas de pielonefrite aguda, tumor intra-abdominal em relação com o sistema urológico ou abdómen agudo, o que motivou a referenciação a serviço de urgência tendo em vista a reavaliação analítica e imagiológica abdominal.

O utente foi reavaliado no mesmo dia em contexto de urgência, sendo que os resultados analíticos foram sobreponíveis aos anteriores. Do ponto de vista imagiológico realizou ecografia abdominal, que mostrou o rim esquerdo completamente substituído por bolsas hidronefróticas de conteúdo não puro e bexiga pouco preenchida, mas de conteúdo puro. Posto isto, decidiu-se complementar esta avaliação com tomografia computorizada urológica, que confirmou rim esquerdo consistindo em volumosas bolsas hidronefróticas com 17 por $15 \mathrm{~cm}$ de dimensão, encontrando-se parênquima renal apenas residual desse lado (Figuras 2 e 3). O bacinete ipsilateral media $11 \mathrm{~cm}$ de diâmetro 


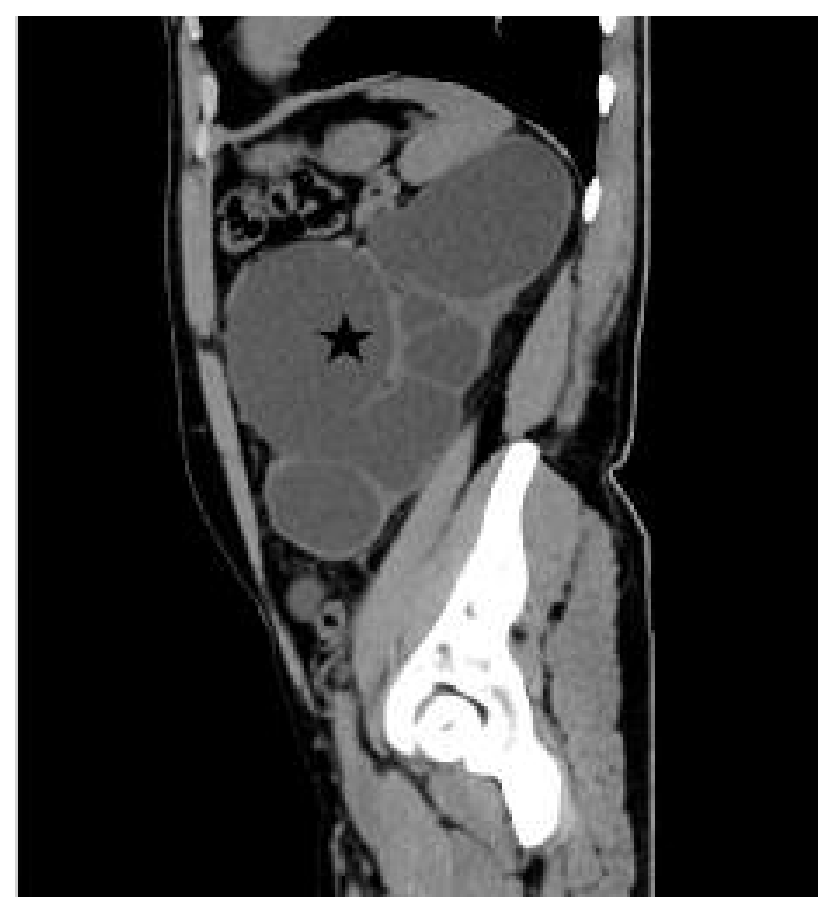

Figura 2. Corte sagital da tomografia computorizada urológica realizada pelo utente. Verifica-se a extensa hidronefrose com parênquima renal residual (estrela).

sem que se observasse ureter a jusante, assim como também não se verificou existência de lesão a causar obstrução. O aparelho renal à direita não evidenciou alterações. Assim, firmou-se o diagnóstico de síndrome da junção pielo-ureteral à esquerda. Posto isto, decidiu-se pelo internamento para cateterização ureteral do lado afetado, procedimento que decorreu sem intercorrências. Realizou ainda urocultura da urina do rim esquerdo que não apresentou crescimento de patógenos, mas a análise do sedimento urinário apresentou abundantes leucócitos e células de descamação. Assim, teve alta ao fim de quatro dias, aguardando renograma em ambulatório e medicado com flavoxato 200mg três vezes por dia.

Passados cinco dias da alta de internamento hospitalar agendou nova consulta em cuidados de saúde primários, onde informou sobre o internamento e afirmou ainda alguma dor abdominal residual, apesar de evolução bastante favorável da sintomatologia. Após explorar eventuais incertezas e dúvidas referentes ao diagnóstico e processo terapêutico futuro concluiu-se que o utente estava esclarecido e a lidar bem com o proces-

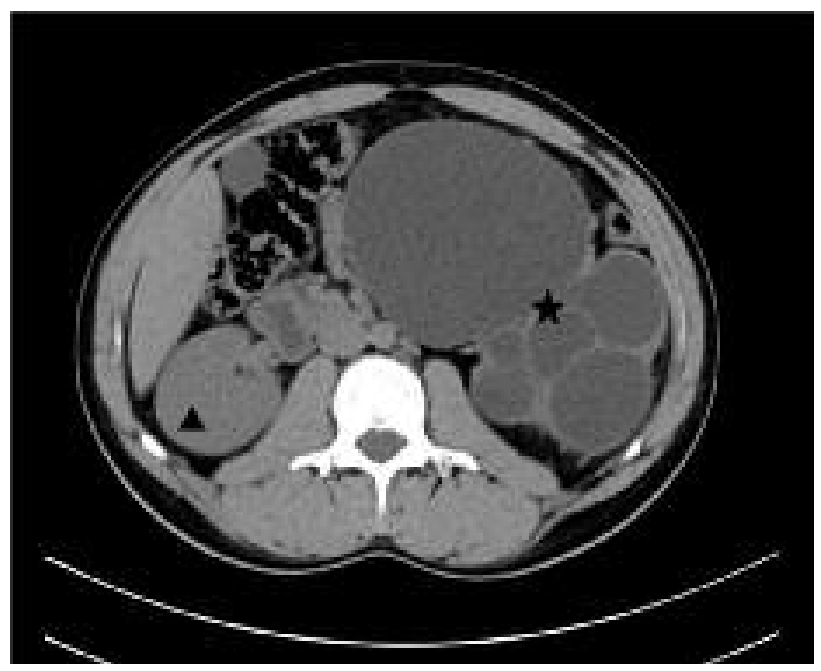

Figura 3. Corte transversal da tomografia computorizada realizada pelo utente. Verifica-se a extensa hidronefrose à esquerda, com parênquima renal residual (estrela), assim como o rim direito sem alterações (triângulo).

so patológico. Prorrogou-se o período definido no certificado de incapacidade temporária e agendou-se consulta programada para continuação dos cuidados.

Passadas algumas semanas realizou renograma, que confirmou função renal residual à direita, pelo que se propôs nefrectomia em consulta de urologia, a qual, à data da redação deste relato de caso, o utente se encontra a aguardar.

\section{COMENTÁRIO}

Na vigência de hematúria macroscópica no jovem adulto, a infeção do trato urinário e a litíase renal devem ser diagnósticos a ter em conta, uma vez que são as suas causas mais frequentes. ${ }^{7-8}$ Não obstante, do diagnóstico diferencial de litíase renal faz parte a síndrome da junção pielo-ureteral, para além da infeção do trato urinário (incluindo pielonefrite aguda, cistite e uretrite), peritonite, rotura de aneurisma da aorta, hérnia inguinal e lombalgia. ${ }^{9}$ Ao passo que os últimos puderam ser excluídos com a avaliação clínica e analítica realizada durante o primeiro episódio de urgência, a síndrome da junção pielo-ureteral necessitava de um exame de imagem adequado para a sua identificação.

Perante a suspeita de cólica renal, a tomografia computorizada renal sem contraste é identificada por várias sociedades científicas como o gold standard para o 
diagnóstico de litíase renal. ${ }^{10-12}$ Permite ainda, caso não se confirme patologia obstrutiva litiásica, a identificação de outras possíveis causas de dor abdominal. ${ }^{10} \mathrm{O}$ uso de ecografia como primeiro método de imagem é aceitável quanto se tratam de crianças ${ }^{11}$ ou como método de avaliação primária, com realização de tomografia computorizada subsequente para confirmação do diagnóstico. ${ }^{12}$ Uma recomendação europeia recente defende a realização de tomografia computorizada renal num período não superior a 14 horas em doentes com suspeita de cólica renal sem outras complicações. ${ }^{10}$ É ainda recomendada a avaliação imagiológica imediata caso se verifique febre, rim único ou dúvida no diagnóstico. ${ }^{10,12}$ Este fator realça ainda mais a importância de um exame objetivo correto como fator decisor de estudo complementar. Mesmo com tempos de consulta reduzidos, que se podem apresentar como obstáculo, um exame objetivo realizado de forma cuidadosa permite, muitas vezes, levantar hipóteses de diagnóstico importantes e decidir sobre a necessidade de tratamento urgente. Em contexto de urgência, a elevada exigência e a escassez de recursos que frequentemente impera podem apresentar-se também como obstáculos à realização de uma consulta eficaz e eficiente, dada a sua relação com o burnout $t^{13} \mathrm{e}$ a eventual associação deste com uma menor qualidade dos cuidados de saúde. ${ }^{14}$

Tendo em conta que a tomografia computorizada realizada não identificou nenhuma estrutura que pudesse justificar a obstrução, é possível que este caso de síndrome da junção pielo-uretral se configure de etiologia congénita, uma vez que o volume da hidronefrose e o grau de involução do parênquima renal sugerem um longo período de evolução. ${ }^{4}$ Do ponto de vista da fisiopatologia, a apresentação tardia que se verificou neste caso pode justificar-se com uma evolução muito insidiosa da obstrução, condicionando o aumento muito gradual do volume de urina retido no aparelho renal, permitindo a adaptação dos tecidos do bacinete ao elevado volume de forma indolor. Geralmente estas alterações tornam-se sintomáticas por intercorrência de outras doenças como, por exemplo, a infeção do trato urinário, que não se chegou a confirmar neste caso. ${ }^{15}$

De um ponto de vista mais centrado no utente, é importante considerar que passará a ser um doente jovem com rim único, pelo que nas próximas consultas será importante gerir as suas expectativas e da sua família, nomeadamente informando, eventualmente desmistificando e ainda planeando a vigilância futura. Por outro lado, este caso acaba por evidenciar a longitudinalidade dos cuidados em medicina geral e familiar. Com efeito, é um médico de família da unidade do utente que orienta de forma adequada o processo diagnóstico, promovendo o desenrolar de um procedimento terapêutico mais apropriado e termina gerindo a convalescença e as necessidades adicionais do doente. Realça ainda a importância da confirmação dos diagnósticos prévios, que algumas vezes acompanham os utentes, assim como a necessidade de confirmar as queixas e sinais apresentados pelos mesmos.

Tendo em conta a implementação de programas de vigilância da gravidez a nível nacional, que incluem a realização de ecografia obstétrica periódica em associação com a cada vez maior qualidade dos aparelhos de ecografia, é expectável que os diagnósticos de síndrome da junção pielo-ureteral congénita em idade adulta se tornem cada vez mais raros. Com efeito, a maioria dos casos deverá ser detetada ainda durante o período fetal e orientada durante a infância ou adolescência. ${ }^{4-6}$

Em suma, este caso é um exemplo de patologia caracteristicamente pediátrica que se pode apresentar em idade adulta, ilustra a importância da confirmação dos diagnósticos prévios e da validação das queixas do utente e pode realçar a necessidade de condições adequadas para a realização da consulta, quer em ambiente de urgência quer em ambiente de cuidados de saúde primários.

\section{AGRADECIMENTOS}

Agradeço o apoio de toda a equipa da USF Amato Lusitano e a ajuda da Enf. Eva Belinha.

\section{REFERÊNCIAS BIBLIOGRÁFICAS}

1. Hashim $\mathrm{H}$,Woodhouse CR. Ureteropelvic junction obstruction. Eur Urol Suppl. 2012;11(2):25-32.

2. González R, Schimke CM. Ureteropelvic junction obstruction in infants and children. Pediatr Clin North Am. 2001;48(6):1505-18.

3. Baskin LS. Congenital ureteropelvic junction obstruction. UpToDate [Internet]; 2019 [updated 2020 Oct 28; cited 2019 May 23]. Available from: https://www.uptodate.com/contents/congenital-ureteropelvicjunction-obstruction

4. Krajewski W, Wojciechowska J, Dembowski J, Zdrojowy R, Szydełko T. Hydronephrosis in the course of ureteropelvic junction obstruction: An underestimated problem? Current opinions on the pathogenesis, diag- 
nosis and treatment. Adv Clin Exp Med. 2017;26(5):857-64.

5. Franco S, Carvalho G, Antunes A, Brito M, Morais C, Barros JS. História natural da dilatação pielocalicial pré-natal [Natural history of fetal pyelocaliectasia]. Acta Med Port. 2005;18(3):169-76. Portuguese

6. Lam JS, Breda A, Schulam PG. Ureteropelvic junction obstruction. J Urol. 200721;177(5):1652-8.

7. Elton TJ, Roth CS, Berquist TH, Silverstein MD. A clinical prediction rule for the diagnosis of ureteral calculi in emergency departments. J Gen Intern Med. 1993;8(2):57-62.

8. Record No. T909566 - Gross hematuria: approach to the adult. DynaMed [Internet]; 2018 [updated 2018 Nov 30; cited 2019 May 23]. Available from: https://www.dynamed.com/topics/dmp AN T909566

9. Record No. T114904 - Nephrolithiasis in adults. DynaMed [Internet]; 2018 [updated 2018 Nov 30; cited 2019 May 23]. Available from: https://www.dynamed.com/topics/dmp AN T114904

10. Tsiotras A, Smith RD, Pearce I, O'Flynn K, Wiseman O. British Association of Urological Surgeons standards for management of acute ureteric colic. J Clin Urol. 2018;11(1):58-61.

11. Fulgham PF, Assimos DG, Pearle MS, Preminger GM. Clinical effectiveness protocols for imaging in the management of ureteral calculous disease: AUA technology assessment. J Urol. 2013;189(4):1203-13.

12. Turk C, Skolarikos A, Neisius A, Petřík A, Seitz C, Thomas K, et al. Urolithiasis [homepage]. Arnhem: European Association of Urology; 2019. ISBN 9789492671042. Available from: https://uroweb.org/guideline/urolithiasis/
13. Marôco J, Marôco AL, Leite E, Bastos C, Vazão MJ, Campos J. Burnout in Portuguese healthcare professionals: an analysis at the national level. Acta Med Port. 2016;29(1):24-30.

14. Dewa CS, Loong D, Bonato S, Trojanowski L. The relationship between physician burnout and quality of healthcare in terms of safety and acceptability: a systematic review. BMJ Open. 2017;7(6):e015141.

15. Zeidel ML, O'Neill WC. Clinical manifestations and diagnosis of urinary tract obstruction and hydronephrosis. UpToDate [Internet]; 2019 [updated May 15; cited 2019 May 23]. Available from: https://www.uptodate.com/contents/clinical-manifestations-and-diagnosis-of-urinarytract-obstruction-and-hydronephrosis

\section{CONFLITO DE INTERESSES}

O autor declara não ter quaisquer conflitos de interesse.

\section{FINANCIAMENTO}

O autor declara não ter recebido qualquer financiamento.

ENDEREÇO PARA CORRESPONDÊNCIA

Samuel Gomes

E-mail: samuelmtgomes@outlook.pt

https://orcid.org/0000-0002-5134-3359

Recebido em 07-11-2019

Aceite para publicação em 07-04-2020

\section{ABSTRACT}

\section{YOUNG ADULT'S HAEMATURIA: AN URETEROPELVIC JUNCTION OBSTRUCTION CLINICAL CASE}

Introduction: The ureteropelvic junction obstruction syndrome is more frequent at paediatric ages. It consists of a urinary tract obstruction that results from a partial or total intermittent blockage of the urine flow at the ureteropelvic junction. It is the most common cause of hydronephrosis in the prenatal period and, for young adults (18 to 24 years old), the hospitalization rate for this cause was 0.7 per 100,000 in 2000, in the United States of America.

Case description: A 21-year-old man, without any relevant past medical or familial history, resorted to the emergency department following pain referred to the left flank and haematuria that started on the same day. There, he was treated symptomatically and was discharged for ambulatory assessment of renal calculi by his family practitioner. Three days later, he scheduled a consultation in his primary care unit. The assessment of the patient was that he maintained the symptoms he had previously, as well as a bilateral absence of costovertebral angle tenderness and an abdominal mass, approximately 15 by $20 \mathrm{~cm}$ in size, elastic in consistency and located in both left abdominal quadrants. With such findings, he was again referred to the emergency department for urgent revaluation. There, a computed tomography scan of the urologic system was taken, revealing extensive hydronephrosis that substituted the left kidney, without apparent obstruction, in accordance with the diagnosis of ureteropelvic junction obstruction syndrome. The patient was hospitalized for ureteral catheterization and left renal function evaluation. Commentary: In the presence of unilateral flank pain with haematuria it raises the suspicion for renal calculi. However, other causes should not be unaddressed, mainly in the presence of less typical presentations of disease. This clinical case reinforces the notion that this paediatric condition can be diagnosed in adult patients, but it is infrequent. In this case, the careful physical evaluation and validation of the patient's complaints by the family practitioner were key aspects for an adequate orientation.

Keywords: Haematuria; Ureteropelvic junction obstruction syndrome; Young adult; Hydronephrosis. 